\title{
TURBULENCE INTENSITY SIMILARITY LAWS FOR TURBULENT BOUNDARY LAYERS
}

\author{
Ivan Marusic \\ Gary J. Kunkel \\ Department of Aerospace Engineering and Mechanics \\ University of Minnesota \\ Minneapolis, MN 55455 \\ marusic@umn.edu, kunkel@aem.umn.edu
}

\begin{abstract}
Turbulence intensity similarity laws, which are based on the attached eddy hypothesis, are tested with new high Reynolds number data obtained in the atmospheric surface layer. The existing formulation for the streamwise component was until now restricted to the log region and above. A new extended version is proposed that applies across the entire boundary layer for smooth wall flows and explains why the nearwall mixed scaling proposed by DeGraaff and Eaton, 2000 appears to be successful.
\end{abstract}

Keywords: Turbulence intensity similarity, atmospheric surface layer, high Reynolds number, attached eddy hypothesis

\section{Introduction}

Data obtained in the surface layer of the atmospheric boundary layer are used to analyze turbulence intensity similarity laws. Historically, the streamwise turbulence intensity is believed to be independent of Reynolds number when scaled with inner-flow variables (the friction velocity, $U_{\tau}$, and the kinematic viscosity) in the entire wall region (viscous sublayer, buffer layer and logarithmic layer) of the turbulent boundary layer. This view is supported by Mochizuki and Nieuwstadt, 1996 and is also used in many computational turbulence models. Recently DeGraaff and Eaton, 2000 have suggested a new mixed scaling that incorporates both $U_{1}$ (freestream velocity) and $U_{\tau}$ and have shown experimental results that support this scaling in the inner-wall region (sublayer and buffer layer). Metzger et al., 2001 have verified this scaling using experimental data from the laboratory and the atmosphere.

Marusic et al., 1997 (referred to here on as MUP) proposed a similarity formulation that is valid in the entire region above the buffer layer 
and that is dependent on Reynolds number. Their formulation is based on the scaling arguments of the power spectral densities and is consistent with Townsend's attached eddy hypothesis. It was found to accurately describe the turbulence intensity over a large range of Reynolds numbers $\left(R e_{\tau} \leq 10^{4}\right)$, however, it was not compared to experimental data obtained at very high Reynolds numbers. To further test the formulations at high Reynolds numbers several experiments have been conducted at the SLTEST (Surface Layer Turbulence and Environmental Science Test) facility located on the western Utah salt flats. The MUP formulation is then extended to apply across the entire boundary layer for smooth wall flow. The extended formulation is compared with laboratory and atmospheric data and is consistent with the suggested mixed scaling of DeGraaff and Eaton, 2000.

\section{Experimental Method}

A detailed description of the SLTEST site and a discussion of the advantages of the site are found in Klewicki et al., 1998. For the data given here the surface roughness was estimated at approximately $10 \mathrm{~mm}$ and the effect of the roughness can be seen in the offset in the mean velocity profiles (not shown). However, by Townsend's Reynolds-numbersimilarity hypothesis this does not effect the turbulence intensities in the outer region of the flow where our measurements are taken. Also, the stability parameter, $z / L$, where $z$ is the wall normal distance and $L$ is the Obukhov length, at the highest wall normal position, $2 \mathrm{~m}$, was positive and less than 0.03. Pahlow et al., 2001 show that the normalized standard deviation of the streamwise velocity component is constant with respect to the stability parameter for positive values of $z / L<0.1$. Therefore, any slight buoyancy effects that might exist are assumed to be negligible. Turbulence intensity profiles are obtained using arrays of cross-wire anemometers that are calibrated at the beginning and end of each run.

\section{Results and Discussion}

Figure 1 shows the streamwise turbulence intensities from several wall normal positions from two data sets obtained in the atmospheric boundary layer $\left(R e_{\tau}=\mathrm{O}\left(10^{6}\right)\right)$. For the data shown the boundary layer thickness was estimated as $200 \mathrm{~m}$ for both data sets (solid symbols). For comparison, data from DeGraaff and Eaton, 2000 are also shown (open symbols). The similarity formulation of MUP,

$$
\frac{<u^{2}>}{U_{\tau}^{2}}=B_{1}-A_{1} \ln \left[\frac{z}{\delta}\right]-V_{g}\left[z^{+}, \frac{z}{\delta}\right]-W_{g}\left[\frac{z}{\delta}\right],
$$


for the corresponding Reynolds numbers are shown along with the formulations if the boundary layer thickness was $\pm 50 \%$ of the estimated value. Here $u$ is the streamwise fluctuating velocity component, $\delta$ is the boundary layer thickness, and $<>$ represents a long time average. The similarity formulations of Högström et al., 2002 are also shown. The MUP formulation appears to describe the data at high Reynolds numbers well. The wall-normal turbulence intensities and the corresponding similarity formulations (Hafez, 1991)

$$
\frac{<w^{2}>}{U_{\tau}^{2}}=A_{3}-B_{3} \frac{z}{\delta}-V\left[z^{+}\right]
$$

are shown in figure 2. Strictly the formulations are only valid for $z^{+}>100$ and $z / \delta<0.15$, however, they have been extended across the entire layer (dashed portions).

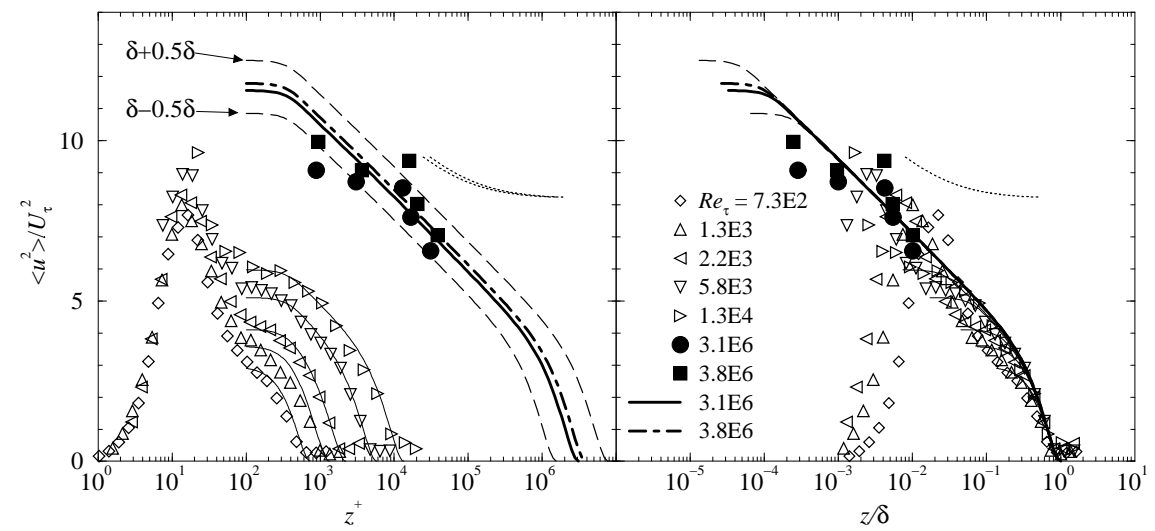

Figure 1. Streamwise turbulence intensities. Solid and dot-dashed lines are the MUP similarity formulation and the dotted lines are the Högström et al., 2002 formulation.

Figure 3 shows the streamwise, wall-normal, and co-spectra with inner-flow scaling. The spectra are consistent with most of the expected spectral scaling laws described in Marusic and Perry, 1995 and with the incomplete similarity hypothesis described by Morrison et al., 2002. For instance, a considerably shorter -1 law is obtained for $\Phi_{11}$ than would be expected by complete similarity (Perry et al., 1986).

\section{Extended Formulation}

While the MUP formulation appears to describe the high Reynolds number data well, it is only applicable in the log region and above. To extend this formulation for smooth walls a blending of scaling regions is 


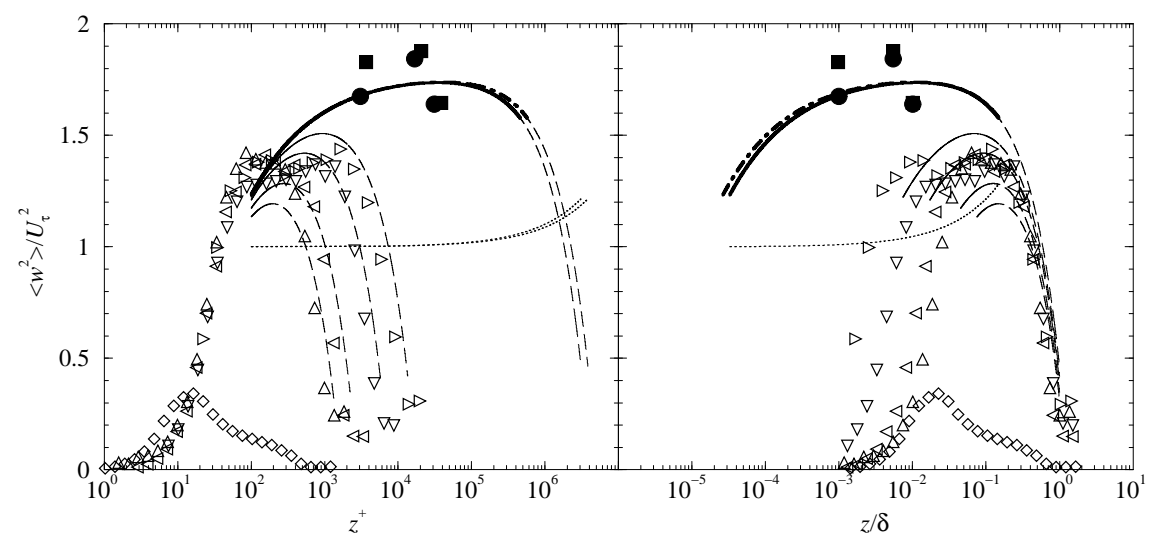

Figure 2. Wall-normal turbulence intensities. Solid and dot-dashed lines are the Hafez, 1991 similarity formulation, other symbols as in figure 1.
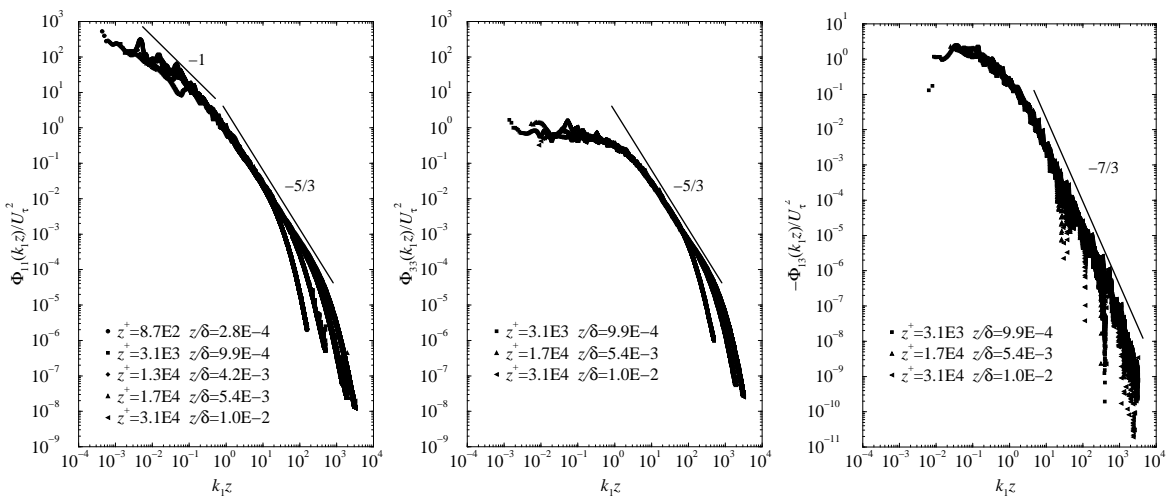

Figure 3. Streamwise, wall-normal and co-spectra with inner-flow scaling in the logarithmic region of the atmospheric surface layer.

considered. The outer part of the boundary layer, $z^{+}>150$, is described by the existing MUP formulation (Eq. 1) that is valid for smooth and rough wall boundary layers. The inner part of the layer $z^{+}<30$ is described by

$$
\frac{<u^{2}>}{U_{\tau}^{2}}=f_{1}\left[z^{+}\right] f_{T}\left[z^{+}, R e_{\tau}\right]
$$

where

$$
f_{1}\left[z^{+}\right]=\frac{0.16\left(z^{+}\right)^{2}}{\left(1+a_{1}\left(z^{+}\right)^{2}\right)^{1 / 2}\left(1+\left(a_{2} z^{+}\right)^{2 a_{3}}\right)^{1 / 2}}
$$

is a empirical curve fit of moderate Reynolds number data valid only for smooth wall boundary layers. Here $f_{T}$ is a simple scaling function that 
is linear in $\ln z^{+}$with a slope proportional to the increase (or decrease) in Reynolds number from the original logarithmic curve fit. These two regions are then blended with a cubic fit between $30<z^{+}<150$. Here $a_{1}=0.008, a_{2}=0.115$, and $a_{3}=1.6$.

The extended formulation with laboratory and atmospheric data can be seen in figure 4a. The extended formulation fits the laboratory data well and suggests that the peak in the turbulence intensity does increase with increasing Reynolds number when scaled with inner-flow variables. The peak in the turbulence intensity, scaled with the friction velocity, as a function of Reynolds number is shown in figure $4 \mathrm{~b}$. The functional form that results from the extended similarity formulation is very similar to the empirical curve fit given by Metzger et al., 2001.

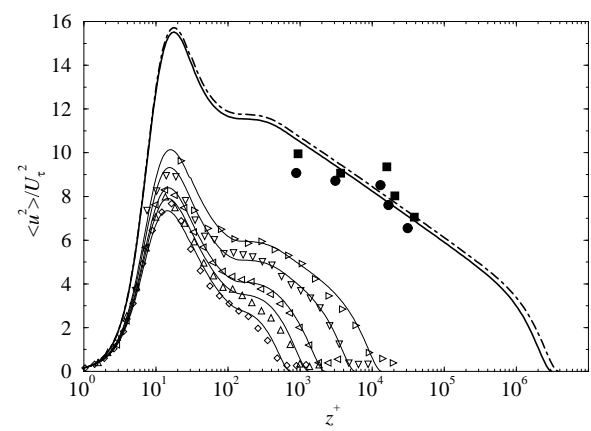

Figure 4a. Extended similarity formulations. Symbols in figure 1.

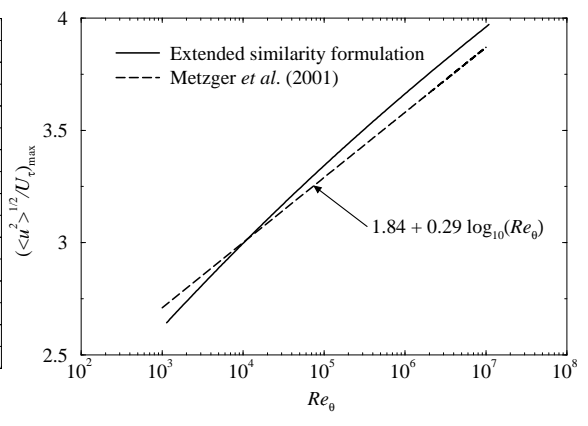

Figure $4 b$. Peak in streamwise turbulence intensity.

Figure 5 shows the extended formulation, laboratory data and atmospheric data with mixed scaling. The extended formulation seems to explain the results of DeGraaff and Eaton, 2000 and why mixed scaling appears to work well.

\section{Conclusion}

An extended similarity formulation has been developed to describe the streamwise turbulence intensity across an entire smooth wall zero pressure gradient turbulent boundary layer. The extended formulation has been compared to moderate and high Reynolds number data obtained in the laboratory and atmospheric boundary layer respectively. The extended formulation is seen to describe the data well and is consistent with the mixed scaling suggested by DeGraaff and Eaton, 2000. 


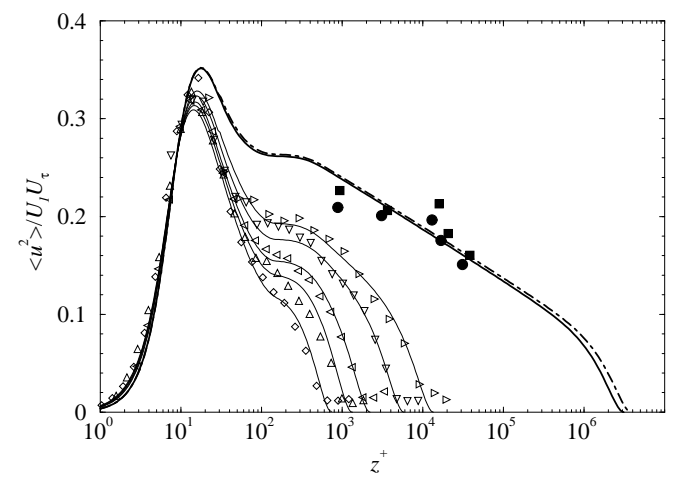

Figure 5. Extended similarity formulations. Symbols in figure 1.

\section{References}

DeGraaff, D. B. and Eaton, J. K. (2000). Reynolds-number scaling of the flat-plate turbulent boundary layer. J. Fluid Mech., 422:319-346.

Hafez, S. H. M. (1991). The structure of accelerated turbulent boundary layers. PhD thesis, University of Melbourne, Australia.

Högström, U., Hunt, J. C. R., and Smedman, A. (2002). Theory and measurements for turbulence spectra and variances in the atmospheric neutral surface layer. Boundary-Layer Meteorology, 103:101-124.

Klewicki, J. C., Foss, J. F., and Wallace, J. M. (1998). High Reynolds number $\left[R_{\theta}=\right.$ $\mathrm{O}\left(10^{6}\right)$ ] boundary layer turbulence in the atmospheric surface layer above Western Utah's salt flats. In Donnelly, R. J. and Sreenivasan, K. R., editors, Flow at UltraHigh Reynolds and Rayleigh Numbers, pages 450-466. Springer.

Marusic, I. and Perry, A. E. (1995). A wall-wake model for the turbulence structure of boundary layers. Part 2. Further experimental support. J. Fluid Mech., 298:389407.

Marusic, I., Uddin, A. K. M., and Perry, A. E. (1997). Similarity law for the streamwise turbulence intensity in zero-pressure-gradient turbulent boundary layers. Phys. Fluids, 9(12):3718-3726.

Metzger, M. M., Klewicki, J. C., Bradshaw, K. L., and Sadr, R. (2001). Scaling the near-wall axial turbulent stress in the zero pressure gradient boundary layer. Phys. Fluids, 13(6):1819-1821.

Mochizuki, S. and Nieuwstadt, F. T. M. (1996). Reynolds-number-dependence of the maximum in the streamwise velocity fluctuations in wall turbulence. Exp. Fluids, $21: 218-226$.

Morrison, J. F., Jiang, W., McKeon, B. J., and Smits, A. J. (2002). Reynolds number dependence of streamwise velocity spectra in turbulent pipe flow. Physical Review Letters, 88(21):214501-1 - 4 .

Pahlow, M., Parlange, M. B., and Porté-Agel, F. (2001). On Monin-Obukhov similarity in the stable atmospheric boundary layer. Boundary-Layer Meteorology, 99:225-248.

Perry, A. E., Henbest, S. M., and Chong, M. S. (1986). A theoretical and experimental study of wall turbulence. J. Fluid Mech., 165:163-199. 


\section{University Library}

\section{- M M N E R VA A gateway to Melbourne's research publications}

Minerva Access is the Institutional Repository of The University of Melbourne

Author/s:

Marusic, l;Kunkel, GJ

Title:

Turbulence intensity similarity laws for turbulent boundary layers

Date:

2004-01-01

Citation:

Marusic, I. \& Kunkel, G. J. (2004). Turbulence intensity similarity laws for turbulent boundary layers. Smits, AJ (Ed.). IUTAM Symposium on Reynolds Number Scaling in Turbulent Flow, (1), 74, pp.17-22. SPRINGER.

Publication Status:

Published

Persistent Link:

http://hdl.handle.net/11343/33512 